\title{
Medical Image of the Week: Tracheobronchial Foreign Body Aspiration
}

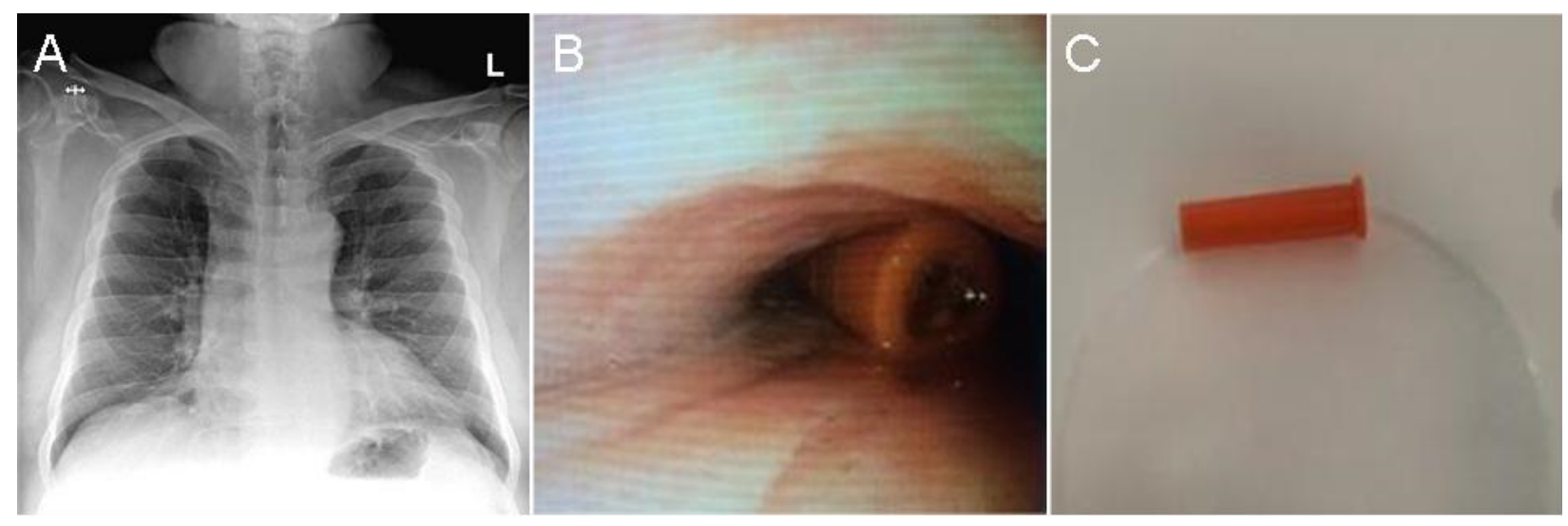

Figure 1. Panel A: The chest $\mathrm{x}$-ray failed to show the aspirated foreign body. Panels $B$ and C: Flexible bronchoscopy was performed and the insulin syringe cap was visualized in the right mainstem bronchus and retrieved with forceps.

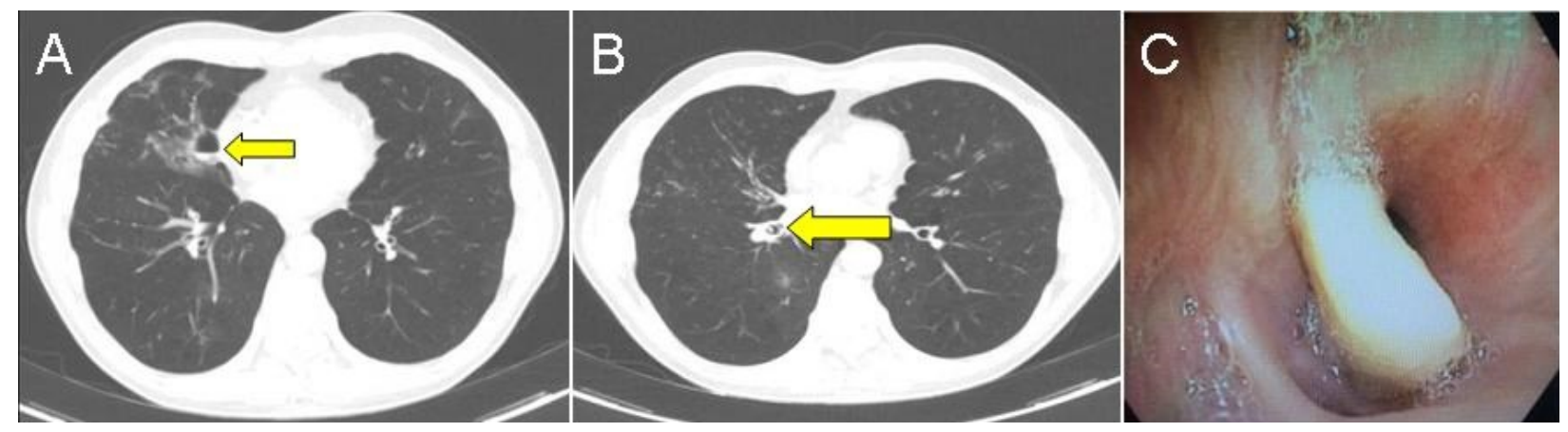

Figure 2. Panel A: CT chest shows interval development of ground glass opacities and air fluid level in the right middle lobe (arrow). Panel B: The foreign body is visualized in the right lower lobe bronchus as an endobronchial-filling defect (arrow). Panel C: Flexible bronchoscopy was performed and cashew piece was retrieved with suction.

Case 1 (Figure 1) is a 58-year-old man who accidentally inhaled his insulin syringe cap while swinging on his recliner with the cap perched in his mouth. He developed a dry irritating cough. On exam he had mild stridor in the upper airways and bilateral wheezing. The insulin cap was visualized by bronchoscopy in the right mainstem bronchus and retrieved with forceps.

Case 2 (Figure 2) is a 65-year-old man with chronic dysphagia and poor dentition who choked on a cashew. It took repeated coughing attempts to produce the cashew, but it was unclear whether the entire content was cleared. He then developed non-massive hemoptysis that persisted for 2 weeks. Thoracic CT showed ground glass opacities and 
an air fluid level in the right middle lobe. The foreign body was visualized in the right lower lobe bronchus as an endobronchial-filling defect. Bronchoscopy revealed a cashew piece in the right lower lobe bronchus. Forceps trials failed due to fragility of the foreign body, which was ultimately retrieved with scope suction.

Rigid bronchoscopy is the gold standard for diagnosis and management of tracheobronchial foreign body aspiration, but flexible bronchoscopy is another accepted method that is also more comfortable for the patient (1). Virtual bronchoscopy is a noninvasive procedure that can assist with localizing the foreign body and may have a role to play in follow-up assessment of airway patency (2). Pneumonia and atelectasis are common complications. Less common complications include bronchiectasis, bronchostenosis, hemoptysis, tracheal perforation, pneumomediastinum, and even cardiopulmonary arrest (3). Tracheal foreign bodies pose more danger than bronchial foreign bodies; in such cases the foreign body should be pushed to distal airways, crumbled if it is organic, and then extracted (1).

Khushboo Goel, MD ${ }^{1}$, Huthayfa Ateeli, MBBS ${ }^{2}$, Joshua Dill, DO2, Dena L'Heureux MD ${ }^{3}$

${ }^{1}$ Department of Internal Medicine, University of Arizona, Tucson, AZ, USA

${ }^{2}$ Department of Internal Medicine, Division of Pulmonary, Critical Care, Sleep, and Allergy Medicine, University of Arizona, Tucson, AZ, USA

${ }^{3}$ Department of Internal Medicine, Division of Pulmonary, Critical Care, and Sleep Medicine, Southern Arizona VA Health Care System, Tucson, AZ, USA

\section{References}

1. Altunas $B$, Aydin $Y$, Eroglu $A$. Foreign bodies in trachea: a 25 year experience. Eurasian J Med. 2016;48(2):119-123. [CrossRef] [PubMed]

2. Kshatriya RM, Khara NV, Paliwal RP, Patel SN. Role of virtual and flexible bronchoscopy in the management of a case of unnoticed foreign body aspiration presented as a nonresolving pneumonia in an adult female. Lung India. 2016; 33(4):420-423. [CrossRef] [PubMed]

3. Altunas $B$, Aydin Y, Eroğlu A. Complications of tracheobronchial foreign bodies. Turk J Med Sci. 2016;46(3):785-800. [CrossRef] [PubMed] 